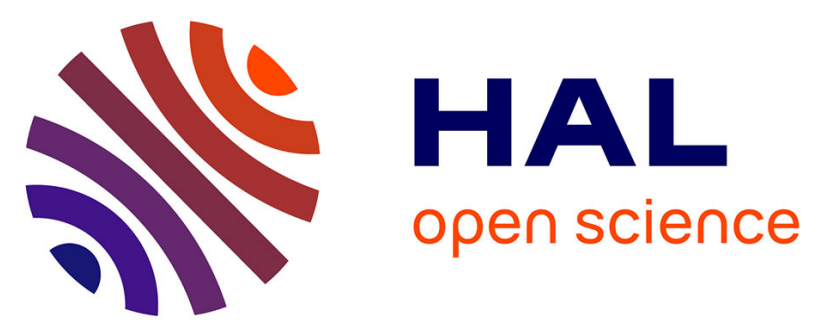

\title{
Effect of refracted light distribution on the photoelastic generation of zero-group velocity Lamb modes in optically low-absorbing plates
}

\author{
Samuel Raetz, Jérôme Laurent, Thomas Dehoux, Daniel Royer, Bertrand
} Audoin, Claire Prada

\section{To cite this version:}

Samuel Raetz, Jérôme Laurent, Thomas Dehoux, Daniel Royer, Bertrand Audoin, et al.. Effect of refracted light distribution on the photoelastic generation of zero-group velocity Lamb modes in optically low-absorbing plates. Journal of the Acoustical Society of America, 2015, 138 (6), pp.3522 3530. 10.1121/1.4936903. hal-01889421

HAL Id: hal-01889421

https://hal-univ-lemans.archives-ouvertes.fr/hal-01889421

Submitted on 9 Oct 2018

HAL is a multi-disciplinary open access archive for the deposit and dissemination of scientific research documents, whether they are published or not. The documents may come from teaching and research institutions in France or abroad, or from public or private research centers.
L'archive ouverte pluridisciplinaire HAL, est destinée au dépôt et à la diffusion de documents scientifiques de niveau recherche, publiés ou non, émanant des établissements d'enseignement et de recherche français ou étrangers, des laboratoires publics ou privés. 


\title{
Effect of refracted light distribution on the photoelastic generation of zero-group velocity Lamb modes in optically low-absorbing plates
}

\author{
Samuel Raetz, ${ }^{1, a)}$ Jérôme Laurent, ${ }^{1}$ Thomas Dehoux, ${ }^{2}$ Daniel Royer, ${ }^{1}$ Bertrand Audoin, ${ }^{2}$ \\ and Claire Prada, ${ }^{1, b}$ \\ ${ }^{1}$ ESPCI ParisTech, CNRS, PSL Research University, Institut Langevin, 1 Rue Jussieu, F-75005, Paris, France \\ ${ }^{2}$ University of Bordeaux, CNRS, Unité Mixte de Recherche 5295, I2M, F-33400, Talence, France
}

(Received 10 June 2015; revised 12 October 2015; accepted 4 November 2015; published online 9 December 2015)

\begin{abstract}
Zero-group velocity (ZGV) Lamb modes are associated with sharp local acoustic resonances and allow, among other features, local measurement of Poisson's ratio. While the thermoelastic generation of Lamb waves in metal plates has been widely studied, the case of materials of low-optical absorption remains unexplored. In materials such as glasses, the generation of bulk elastic waves has been demonstrated to be sensitive to the refracted light distribution. In this paper, a detailed analysis of the effect of light refraction on the laser-based generation of ZGV Lamb modes is presented. Experiments are performed on a bare glass plate without the need for an additional layer for light absorption or reflection. Using an appropriate tilted volume source, it is shown that the laserultrasonic technique allows non-contact measurement of the Poisson's ratio.
\end{abstract}

(C) 2015 Acoustical Society of America. [http://dx.doi.org/10.1121/1.4936903]

$[\mathrm{ANN}]$

Pages: $3522-3530$

\section{INTRODUCTION}

Non-contact characterization of mechanical properties using laser ultrasonic techniques is very attractive. For the last decades, numerous studies have dealt with the thermoelastic generation of Rayleigh and Lamb waves in metal plates. ${ }^{1-3}$ Laser ultrasonic techniques have been applied to mechanical characterization, using broadband generation methods, i.e., laser pulses focused to a point or to a line, ${ }^{4-11}$ or using narrowband generation methods relying on optical interference techniques. ${ }^{12-17}$ Yet in materials of high-optical absorption ${ }^{18}$ such as metals, the photoacoustic source lies on the surface.

The effect of optical absorption on the generation of Lamb waves with a laser source was first studied by Hurley and Spicer for moderately absorbing plates (penetration depth smaller than the plate thickness). ${ }^{19}$ They observed the dependence of $A_{0}$ and $S_{0}$ Lamb mode amplitudes on the optical absorption coefficient. The effect of optical absorption on the generation of ultrasonic bulk waves in optically moderate- to low-absorbing materials was also intensively studied for the last decades ${ }^{20-24}$ but always considering the laser beam to be normal to the surface of the sample. Recently the generation of ultrasonic bulk waves in moderately absorbing glass samples was investigated. ${ }^{25}$ The directivity of longitudinal and transversal waves was analyzed as a function of the laser beam angle of incidence. ${ }^{26}$ However, the effect of refracted light distribution on the generation of Lamb waves is still unexplored.

\footnotetext{
${ }^{a}$ Current address: LUNAM Universités, CNRS, Université du Maine, LAUM UMR-CNRS 6613, Av. O. Messiaen, 72085 Le Mans, France.

b)Electronic mail: claire.prada-julia@espci.fr
}

The propagation of symmetric $(S)$ and antisymmetric (A) Lamb modes, guided by the plate structure, can be represented by a set of dispersion curves giving the angular frequency $\omega$ versus the wave number $k .^{27,28}$ The acoustic energy resulting of the absorption of a laser beam of finite lateral size rapidly flows out of the source area except for nonpropagative Lamb modes. These exotic modes exist at a specific frequency for which the group velocity $V_{g}=\mathrm{d} \omega / \mathrm{d} k$ vanishes, while the phase velocity remains finite. The frequencies of zero-group velocity (ZGV) Lamb modes are slightly lower than cut-off frequencies corresponding to extensional or shear thickness resonances. For example, the first ZGV mode occurs for a specific value $k_{0} d$ of the wave number by plate thickness $(d)$ product corresponding to the junction of $S_{1}$ and $S_{2 b}$ branches, where $b$ stands for the backward wave, ${ }^{29,30}$ and is denoted $S_{1} S_{2}-\mathrm{ZGV}$ mode. ${ }^{31}$ The frequency of the $S_{1} S_{2}-\mathrm{ZGV}$ mode is indeed smaller than the frequency of the first extensional thickness resonance, which is equal to $V_{L} / 2 d$, where $V_{L}$ is the compressional wave velocity.

Experimental studies, performed in the thermoelastic regime on materials with high-optical absorption such as metals, have shown that ZGV modes are associated with sharp local resonances of the plate. ${ }^{27,28}$ For a surface thermoelastic source, the in-plane dipole force efficiently couples to ZGV modes contrary to extensional thickness modes that require out-of-plane force to be efficiently generated. As a consequence, the spectrum of the normal displacement is dominated by ZGV resonances. Except for the first three $\left(S_{0}\right.$, $A_{0}$ and $A_{1}$ ) Lamb modes, the dispersion curves of all higher order modes exhibit a minimum frequency for some Poisson's ratio. ${ }^{31}$

In material science, glasses are interesting as model materials because they offer a good opportunity to vary the 
composition smoothly and thus to obtain a wide range of Poisson's ratio. ${ }^{33}$ Moreover, there is a growing interest in measurements of phase transitions in transparent glassy materials at high temperatures or under extreme pressure conditions. ${ }^{34,35}$ For these reasons, a contactless technique to probe locally Poisson's ratio of transparent materials such as glasses is needed. Numerous characterization methods already allow measurement of the Poisson's ratio. ${ }^{36}$ These methods are typically based on mechanical probing of the sample, vibrations, ultrasound, or $\mathrm{x}$ rays. Some of these techniques have in common the necessity of a mechanical contact with the probed material, which can affect the measurement and mainly be an issue under extreme temperature/pressure conditions. To overcome this limitation, laser ultrasonic techniques is a very attractive solution. ${ }^{8,37-43}$

For glass plates, Poisson's ratio $\nu$ usually ranges from 0.17 to 0.27 , and both $S_{1} S_{2^{-}}$and $A_{2} A_{3}-\mathrm{ZGV}$ modes exist. ${ }^{31} \mathrm{It}$ was shown that the Poisson's ratio can be determined from the ratio of the resonance frequencies of the symmetric $S_{1} S_{2^{-}}$ and antisymmetric $A_{2} A_{3}-Z G V$ modes. ${ }^{32}$ The generation of ZGV Lamb mode resonances was first observed in fused silica by adding a thin aluminum layer, ${ }^{32}$ allowing photoacoustic transduction to occur at the surface of the silica. In this paper, we propose to study the thermoelastic generation of ZGV Lamb modes in bare glass plates, i.e., without any metallic coating. In such low-absorbing media, the optical penetration depth is larger than the sample thickness, implying that the acoustic source is distributed along the light path from the front to rear surface of the sample. According to symmetry, the generation of antisymmetric Lamb modes at normal incidence of the laser beam is thus reduced. To overcome this feature limiting Poisson's ratio measurement, we propose to tilt the laser beam to break the source symmetry.

In the following, the model described in Ref. 25 is used to compute the generation of elastic waves due to a tilted volume thermoelastic source and to analyze the effect of refracted light distribution on ZGV Lamb mode generation. This model is based on a semi-analytical solution of Maxwell's equations together with the heat diffusion and elastic wave equations in the spatiotemporal Fourier domain. The generation of the first two ZGV Lamb modes $\left(S_{1} S_{2}\right.$ and $A_{2} A_{3}$ ) is studied in detail, thus providing the optimal angle of light incidence to determine the Poisson's ratio. Measurements made on a window glass plate are then presented and illustrate the theoretical predictions.

\section{THEORETICAL BACKGROUND}

The thermoelastic generation of elastic waves due to laser absorption in an optically low-absorbing plate is illustrated in Fig. 1. A pulsed laser source is focused to a line at the front surface $(z=0)$ of a window glass plate. The plate thickness $d=2 h$ is far smaller than the optical penetration depth $\xi$ of the glass. The partial absorption of the light produces local heating all along the light path. The thermal expansion of the heated volume generates elastic waves propagating in the plate: compressional and shear bulk waves, Rayleigh and Lamb waves.

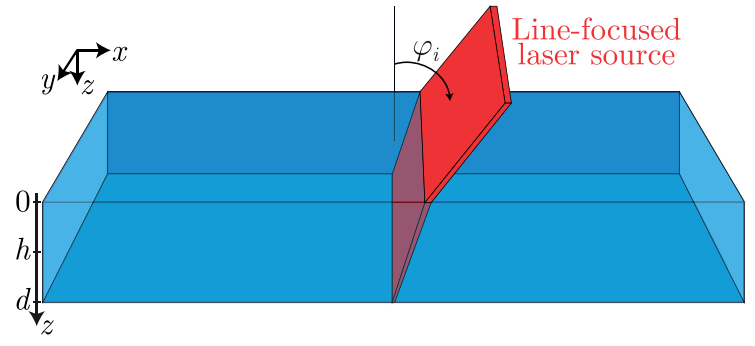

FIG. 1. (Color online) Geometry of the acoustic source resulting of the absorption of a laser beam focused to a line on the surface of a lowabsorbing plate.

The $z$ axis in Fig. 1 stands for the direction normal to the plate surfaces. The laser line direction is parallel to the $y$ axis, and Lamb waves propagate along the $x$ axis. The origin of the $x$ axis coincides with the position where the laser beam emerges of the rear surface of the plate $(z=d)$. Because the thermoelastic source is distributed in the bulk of the plate, the elastic wave generation depends on the angle of incidence $\varphi_{i}$ of the laser beam (Fig. 1). Equations modeling the elastic wave propagation in a plate are now presented.

Because the laser beam is focused to a line, the problem is considered as two dimensional in the $(x, z)$ plane. The displacement field generated by such a source is solution of the acoustic wave equation

$$
\boldsymbol{\nabla} \cdot\left(\underline{\mathbf{C}}: \nabla^{\mathbf{S}} \mathbf{u}\right)-\rho \frac{\partial^{2} \mathbf{u}}{\partial t^{2}}=\mathbf{S},
$$

where $\rho$ is the mass density, $\mathbf{u}$ is the displacement vector, and $\underline{\mathbf{C}}$ is the fourth-order stiffness tensor. The quantity $\nabla^{\mathbf{S}} \mathbf{u}$ is the second-order strain tensor. The source term $\mathbf{S}$ is the divergence of the thermal stresses induced by the laser absorption

$$
\mathbf{S}(x, z, t)=\underline{\lambda} \boldsymbol{\nabla} T(x, z, t),
$$

where $\underline{\lambda}$ is the second-order thermal dilatation tensor and $T$ stands for the temperature rise resulting from the laser absorption. The analytical expressions of optical absorption $\beta\left(\varphi_{r}\right)$ and of temperature $T$ can be calculated from Maxwell's equations and heat equation neglecting thermal diffusion. $^{25}$ Because the reflection coefficient of the laser source at the glass/air interface is less than $4.2 \%$ for angles of incidence smaller than $70^{\circ}$, the optical reflection at the interface $z=d$ can be neglected. The temperature rise $T$ is then given by the following expression:

$$
\begin{aligned}
T(x, z, t)= & \frac{\beta\left(\varphi_{r}\right) I}{\rho C_{p}} \exp \left[-\beta\left(\varphi_{r}\right) z\right] \\
& \times G\left(x \cos \varphi_{r}^{\text {real }}-z \sin \varphi_{r}^{\text {real }}\right) F(t),
\end{aligned}
$$

where $C_{p}$ and $I$ are the specific heat of the material and the transmitted intensity of the laser beam, respectively. The temperature rise decreases exponentially with respect to the depth $z$, accordingly with the absorption $\beta\left(\varphi_{r}\right){ }^{25} \varphi_{r}$ is the complex angle satisfying Snell's law in an optically absorbing medium: $\sin \varphi_{i}=n^{*} \sin \varphi_{r}$ with $n^{*}$ the complex 
index of refraction. As the heat diffusion was neglected, function $F(t)$ is the primitive function of the normalized time distribution of laser intensity. The quantity $\varphi_{r}^{\text {real }}$ in Eq. (3) is the angle of refraction of the laser beam: $\varphi_{r}^{\text {real }}$ $=\sin ^{-1}\left(\sin \varphi_{i} / n\right)$, where $n$ is the real part of $n^{*}$. Assuming a Gaussian distribution, the normalized laser intensity is expressed as $G(x)=(2 / a) \sqrt{\ln 2 / \pi} \exp \left[-4 \ln 2\left(x^{2} / a^{2}\right)\right]$ where $a$ is the full width at half maximum (FWHM).

The elastic wave propagation equation [Eq. (1)] can be solved analytically in the frequency-wavenumber Fourier domain. The spatiotemporal inverse Fourier transform can then be calculated numerically. Hence elastic wave fields generated either by a volume acoustic source or a surface acoustic source, modelled by Eq. (2), are thus computed and the ZGV Lamb mode generation by such sources can be analyzed.

Figure 2 displays the theoretical dispersion curves of Lamb waves propagating in the 2.085-mm-thick window glass used in the experiment of which mechanical properties are listed in Table I. In this plate, two ZGV modes (vertical arrows) are found at frequencies $f_{S_{1} S_{2}}=1.32 \mathrm{MHz}$ and $f_{A_{2} A_{3}}$ $=2.439 \mathrm{MHz}$ and wavenumbers $k_{S_{1} S_{2}}=0.864 \mathrm{~mm}^{-1}$ and $k_{A_{2} A_{3}}=1.019 \mathrm{~mm}^{-1}$.

In Sec. III, it is proposed to compute and to analyze elastic wave fields generated either by a tilted volume acoustic source or by a surface acoustic source. Because the light reflection at $z=d$ is not taken into account, the model is the same as the one exposed in Ref. 25. The surface acoustic source is obtained by setting a very large value for the optical absorption. Important differences in the wave fields for a volume source or a surface source are demonstrated and discussed, leading to a better understanding of the generation of ZGV Lamb modes by the tilted volume source.

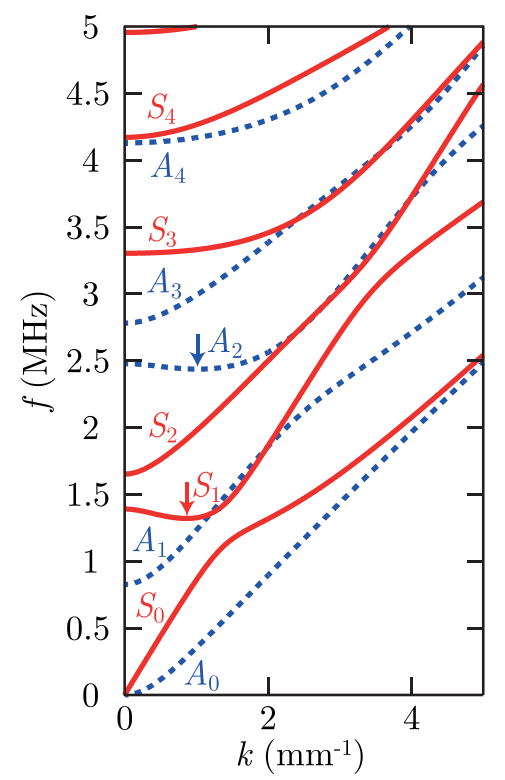

FIG. 2. (Color online) Dispersion curves of the symmetric (solid line) and antisymmetric (dashed line) Lamb modes calculated in a 2.085-mm-thick glass plate. Two ZGV modes (vertical arrows) are found at $f_{S_{1} S_{2}}=1.32 \mathrm{MHz}$ and $f_{A_{2} A_{3}}=2.439 \mathrm{MHz}$, and $k_{S_{1} S_{2}}=0.864 \mathrm{~mm}^{-1}$, and $k_{A_{2} A_{3}}=1.019 \mathrm{~mm}^{-1}$.
TABLE I. Mechanical, thermal, and optical (at $1064 \mathrm{~nm}$ ) properties of the window glass.

\begin{tabular}{lc}
\hline \hline & \\
\hline Thickness $d$ & Value \\
Compressional wave velocity $V_{L}$ & $2.085 \mathrm{~mm}$ \\
Shear wave velocity $V_{T}$ & $5800 \mathrm{~m} \mathrm{~s}^{-1}$ \\
Mass density $\rho$ & $3440 \mathrm{~m} \mathrm{~s}^{-1}$ \\
Specific heat $C_{p}$ & $2500 \mathrm{~kg} \mathrm{~m}^{-3}$ \\
Thermal expansion $\alpha$ & $840 \mathrm{~J}\left(\mathrm{~kg} \mathrm{~K}^{-1}\right.$ \\
Refractive index $n=\Re\left(n^{*}\right)$ & $6.6 \times 10^{-6} \mathrm{~K}^{-1}$ \\
Absorption coefficient $\kappa=\Im\left(n^{*}\right)$ & 1.51 \\
Optical penetration depth $1 / \beta\left(0^{\circ}\right)$ & $4.4 \times 10^{-6}$ \\
\hline \hline
\end{tabular}

\section{EFFECTS OF A TILTED ACOUSTIC SOURCE ON ZGV LAMB MODE GENERATION}

In the following, the semi-analytical model presented in Sec. II is used to compare the elastic wave fields generated either by a surface or a volume acoustic source. Optical divergence is neglected in the optically low-absorbing sample, and the width of the surface and volume acoustic sources are assumed to be equal to the experimental value: $a=150 \mu \mathrm{m}$. The relevant physical properties of the window glass are summarized in Table I.

Figure 3 displays the distribution of the normalized amplitude of the displacement calculated for a 2.085-mm-thick window glass plate at time $t=0.4 \mu \mathrm{s}$ after laser impact for (a) a surface acoustic source and (b) a tilted volume acoustic source $\left(\varphi_{i}=50^{\circ}\right)$. The wave fields generated in both cases at early time after the laser impact are substantially different. They satisfy different symmetries imposed by both the source and the sample geometries. On the one hand, the elastic wave field generated by the surface source is symmetrical with respect to the plane parallel to the $(x, y)$ plane and containing the line source. This plane intersects the $(x, z)$ plane along the vertical line of abscissa $x=d \tan \varphi_{r}^{r e a l}$. On the other hand, the wave field generated by the volume source is symmetrical with respect to the line along $y$ located at the intersection of the median plane $z=h$ with the plane

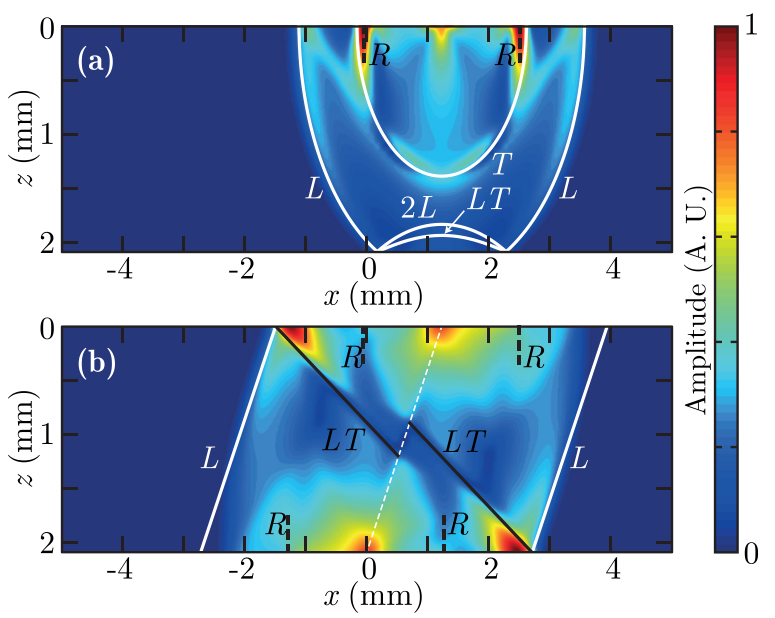

FIG. 3. (Color online) Distribution of the amplitude of the displacement in a 2.085-mm-thick window glass plate at $0.4 \mu \mathrm{s}$ after laser impact for (a) a surface source and (b) a tilted volume source $\left(\varphi_{i}=50^{\circ}\right)$. 
containing the source. This line intersects the $(x, z)$ plane at the symmetry point $x=h \tan \varphi_{r}^{\text {real }}, z=h$.

For the surface acoustic source [Fig. 3(a)], standard circular wavefronts of compressional waves $(L)$, shear waves $(T)$, and waves reflected at the rear surface of the plate, $z=d$, with or without mode conversion ( $L T$ and $2 L$, respectively), are observed. The Rayleigh waves $(R)$ are generated and propagate at the surface $z=0$ only. Conversely, for the volume source distributed along the direction of refracted light propagation [dashed white line in Fig. 3(b)], the generated bulk waves are mainly plane compressional waves $L$ with a propagation direction normal to the refracted light beam. Indeed the volume expansion due to the temperature rise occurs essentially in a direction normal to the direction of refraction. ${ }^{26}$ The $L$ waves are reflected and mode-converted at the free surfaces, giving rise to shear plane waves $L T$ propagating in a direction defined by the Snell's law. Because the source energy is spread over the sample thickness, the energy density remaining in the skin depth of the Rayleigh wave is small. Thus Rayleigh waves $(R)$ are generated with small amplitudes at both surfaces, $z=0$ and $z=d$.

It is expected that in a low-absorbing plate, where the acoustic source is distributed in the volume, the excitation of ZGV Lamb modes occurs differently than for a metallic plate. This effect can be illustrated by analyzing the distribution of the normal displacement a few microseconds after the laser impact when the propagative elastic modes have escaped the thermally stressed zone, while ZGV Lamb mode resonances are established. The normal displacement can be observed in Figs. 4(a) and 4(b) for the surface source and $4(\mathrm{c})$ and $4(\mathrm{~d})$ for the tilted volume source $\left(\varphi_{i}=50^{\circ}\right)$ at time $t=19.22 \mu \mathrm{s}$ for Figs. 4(a) and 4(c) and at time $t=19.44 \mu \mathrm{s}$ for Figs. 4(b) and 4(d). From Fig. 2, at least two ZGV modes are expected, one symmetric $S_{1} S_{2}$ and one antisymmetric $A_{2} A_{3}$. The normal displacement associated with $S_{1} S_{2}$ is an odd function of $z$ with respect to $z=h$, whereas the normal displacement associated with $A_{2} A_{3}$ is an even function of $z{ }^{27}$
So, odd [Figs. 4(a) and 4(c)] and even [Figs. 4(b) and 4(d)] parts of the normal displacement are separately displayed; this is straightforward using the semi-analytical calculation because this separation (odd/even) has been used to solve the linear system of equations. Each map is normalized by its maximum, the color-scale thus refers to normalized values from -1 to 1 . In Figs. 4(a) and 4(b), the arrow indicates the centre of the laser source. In Figs. 4(c) and 4(d), the white line represents the direction of light refraction.

In Figs. 4(a) and 4(c), it appears that the $S_{1} S_{2}-\mathrm{ZGV}$ mode is well established in both cases, at least for $x \in[-10 ; 10]$ $\mathrm{mm}$. Note that the time $t=19.22 \mu \mathrm{s}$, at which the odd component of the normal displacement is displayed, corresponds to $t=25.3 T_{S_{1} S_{2}}$, where $T_{S_{1} S_{2}} \simeq 0.76 \mu \mathrm{s}$ is the period of the $S_{1} S_{2}$ mode. For the surface source, the $S_{1} S_{2}$ symmetric mode is centered on the source center abscissa $x=d \tan \varphi_{r}^{\text {real }}$ [Fig. 4(a)]. However, for the tilted volume source, because the in-depth distribution of the out-of-plane displacement of the $S_{1} S_{2}$ mode is antisymmetrical with respect to $z=h$, the $S_{1} S_{2}$ mode is shifted along the $x$ axis so that the out-of-plane displacement remains symmetrical with respect to the plane of abscissa $x=h \tan \varphi_{r}^{\text {real }}$ [Fig. 4(c)].

Comparing Figs. 4(b) and 4(d) with Figs. 4(a) and 4(c), it appears that the $A_{2} A_{3}-Z G V$ mode is not as well established as the $S_{1} S_{2}$ mode, i.e., three lobes along $x$ are clearly visible for the $A_{2} A_{3}-Z G V$ mode, whereas five lobes covering $20 \mathrm{~mm}$ along $x$ are established for the $S_{1} S_{2}-\mathrm{ZGV}$ mode. Yet, the even component of the displacement is displayed at $t=19.43 \mu \mathrm{s}$, which corresponds to $t=47.4 T_{A_{2} A_{3}}$, where $T_{A_{2} A_{3}} \simeq 0.41 \mu \mathrm{s}$ is the period of the $A_{2} A_{3}$ mode. Because the $S_{1} S_{2}$ mode is better established after 25 periods than the $A_{2} A_{3}$ mode after 47 periods, it seems that the fewer nodes in the depth profile of the mode displacement distribution (one node for $S_{1} S_{2}$ and two nodes for $A_{2} A_{3}$ ), the shorter the resonance settling time. As expected, for the surface source, the mode pattern is symmetrical with respect to the source abscissa $x=d \tan \varphi_{r}^{\text {real }}$ [Fig. 4(b)]. However, for the tilted
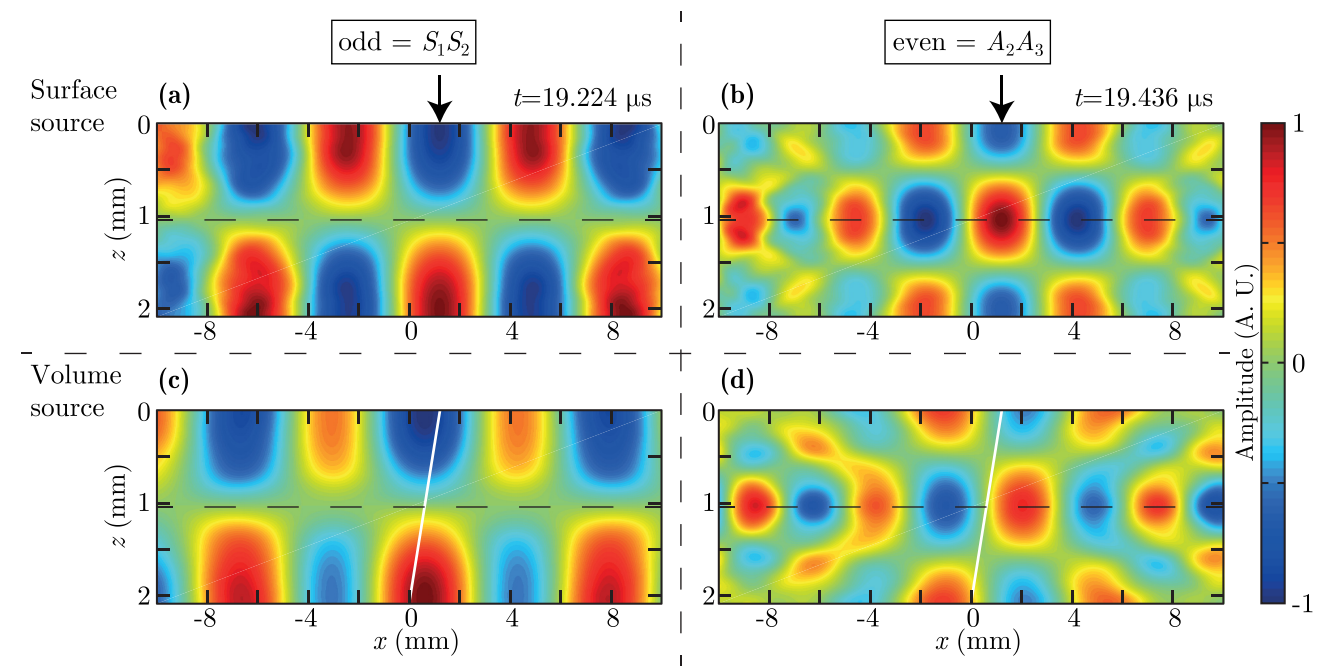

FIG. 4. (Color online) Odd and even components of the normal displacement calculated in a window glass plate for (a) and (b) the surface source and (c) and (d) the tilted volume source $\left(\varphi_{i}=50^{\circ}\right)$ : the odd parts at time $t=19.22 \mu$ s [(a) and (c)] reveal the symmetric $S_{1} S_{2}-\mathrm{ZGV}$ mode, while the even parts at time $t=19.44 \mu \mathrm{s}\left[(\mathrm{b})\right.$ and (d)] reveal the antisymmetric $A_{2} A_{3}-\mathrm{ZGV}$ mode. In (a)-(b), the arrow is pointed on the laser source centre. In (c)-(d), the white line represents the direction of light refraction. 
volume source, because the in-depth distribution of the outof-plane displacement of the $A_{2} A_{3}$ mode is symmetrical with respect to $z=h$, the resulting $A_{2} A_{3}$ distribution is antisymmetrical with respect to the plane of abscissa $x=h \tan \varphi_{r}^{\text {real }}$ [Fig. 4(d)]. Furthermore, a slight asymmetry is observed due to optical absorption.

The dependence on the angle of incidence of the amplitude of the first two ZGV Lamb modes generated by a tilted volume source is now investigated. Using analytic calculations in the spatiotemporal Fourier domain, the amplitude of the spectrum of the normal displacement, denoted $\left|\tilde{U}\left(f, k, \varphi_{i}\right)\right|$, is calculated for both couples: $\left(f_{S_{1} S_{2}}, k_{S_{1} S_{2}}\right)$ and $\left(f_{A_{2} A_{3}}, k_{A_{2} A_{3}}\right)$. Figure 5 displays the value of $\left|\tilde{U}\left(f, k, \varphi_{i}\right)\right|$ as a function of $\varphi_{i}$ for the $S_{1} S_{2}$ mode (solid line) and for the $A_{2} A_{3}$ mode (dashed line) at a constant incident laser energy. The amplitude of the $S_{1} S_{2}$-ZGV resonance decreases with increasing $\varphi_{i}$, while the amplitude of the $A_{2} A_{3}-Z G V$ mode first goes through a maximum for $\varphi_{i}=55.5^{\circ}$. For a better understanding of these dependences versus $\varphi_{i}$, the absorbed laser power $P\left(\varphi_{i}\right)$ is plotted in Fig. 5 (dashed-dotted line). The decay of $P\left(\varphi_{i}\right)$ is mainly due to the increase of the optical reflection coefficient. Thus, the amplitude variation of the $S_{1} S_{2}-\mathrm{ZGV}$ mode is similar to decay of $P\left(\varphi_{i}\right)$. The slight discrepancy can be ascribed to the loss of symmetry of the volume source. Conversely, this loss of symmetry is significantly beneficial for the generation of the antisymmetric $A_{2} A_{3}-\mathrm{ZGV}$ mode. The competition between the enhancement of the $A_{2} A_{3}-\mathrm{ZGV}$ mode and the decrease in absorbed laser power with increasing $\varphi_{i}$ explains the existence of an optimum angle of incidence (Fig. 5).

As each curve is normalized, it is important to note that the absolute amplitude of the $A_{2} A_{3}-\mathrm{ZGV}$ mode always remains lower than the amplitude of the $S_{1} S_{2}-\mathrm{ZGV}$ mode. Indeed even for the optimum angle $\varphi_{i}^{A_{2} A_{3}}$, the amplitude of $S_{1} S_{2}$-ZGV resonance is one order of magnitude larger than the amplitude of $A_{2} A_{3}-Z G V$ resonance. For comparison, at $\varphi_{i}=0^{\circ}$, this ratio is about 150 . At normal incidence, the generation of the $A_{2} A_{3}$ resonance is very small but non-zero. This is due to the exponential decay with $z$ of the laser heating, leading to a slight asymmetry of the source.

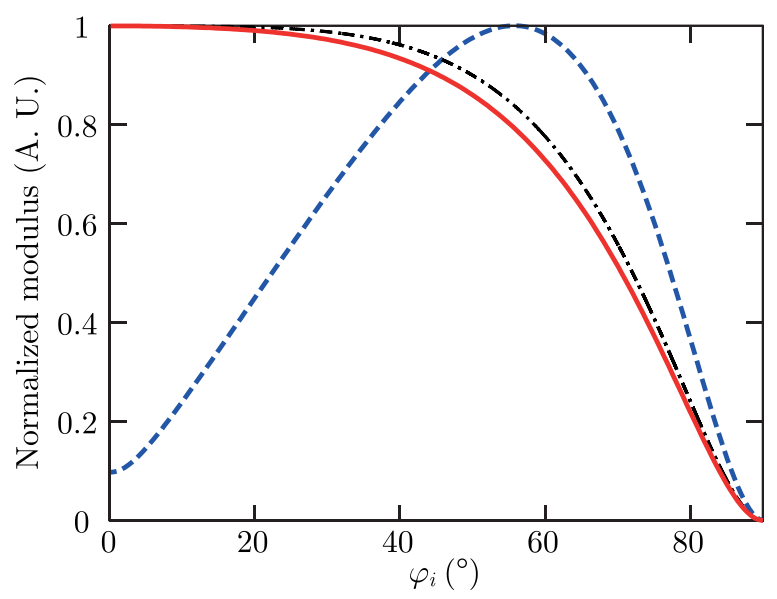

FIG. 5. (Color online) Amplitude $\left|\tilde{U}\left(f, k, \varphi_{i}\right)\right|$ versus $\varphi_{i}$ of $S_{1} S_{2}$ mode (solid line) and $A_{2} A_{3}$ mode (dashed line), absorbed laser power $P\left(\varphi_{i}\right)$ (dasheddotted line). Each curve is normalized with respect to its maximum.
To determine the Poisson's ratio with a single measurement, two $\mathrm{ZGV}$ resonances have to be simultaneously detected. The previous observations suggest two practical points for measurement in bare glass plates: the source laser beam should be tilted to enhance the generation of the $A_{2} A_{3}-$ $\mathrm{ZGV}$ mode, and the detection point has to be chosen such that the normal displacement is significant for both modes. For a tilted volume source, this point is not located at $x=0$ because the ZGV modes are not spatially in phase [Figs. 4(c) and $4(\mathrm{~d})]$. This will be confirmed by the experimental profiles shown in Sec. IV.

\section{EXPERIMENTAL RESULTS AND COMPARISON WITH THEORETICAL PREDICTIONS}

The experimental setup (Fig. 6) was composed of a pulsed Nd:YAG laser with a repetition rate of $100 \mathrm{~Hz}$ (Quantel Centurion) focused with a cylindrical lens to a line of width $\sim 150 \mu \mathrm{m}$ and length $\sim 14 \mathrm{~mm}$ at the front surface $(z=0)$ of a 2.085-mm-thick window glass plate. The laser delivers pulses of $8.5 \mathrm{~ns}$ duration and up to $40 \mathrm{~mJ}$ energy $(20 \mathrm{~mJ}$ in these experiments). The angle of incidence was varied using a motorized goniometer on which the laser source was fixed. Measurements were performed for ten values of $\varphi_{i}: 10^{\circ}, 20^{\circ}$, $30^{\circ}, 40^{\circ}, 45^{\circ}, 50^{\circ}, 55^{\circ}, 60^{\circ}, 65^{\circ}$, and $70^{\circ}$. For each angle of incidence, the distance between the cylindrical lens and the sample was adjusted to ensure that the focusing occurs at the front surface of the sample. Note that the ratio of the FWHM of the laser beam at $z=d$ to the FWHM at $z=0$ was estimated to be less than 1.3, which means that all along the light path, the beam width was very small compared to the plate thickness.

A heterodyne interferometer (SH140, by BMI) was used to measure the normal displacement of the rear surface of the plate without any coating. This was possible because the interferometer beam was focused with a lens having a depth of field $( \pm 0.7 \mathrm{~mm})$ smaller than the plate thickness. Thus the light reflected by the rear surface only was collected by the optics of the interferometer. The interferometer included a band-pass filter operating between $320 \mathrm{kHz}$ and $40 \mathrm{MHz}$. The interferometer to laser source position was scanned along the $x$ axis using a motorized stage from $x=-10$ to $10 \mathrm{~mm}$ with $0.1 \mathrm{~mm}$ steps.

The experimental signals for $\varphi_{i}=50^{\circ}$ are plotted with respect to time $t$ and to the interferometer position $x$ (Fig. 7). Signals are normalized to the maximum amplitude measured

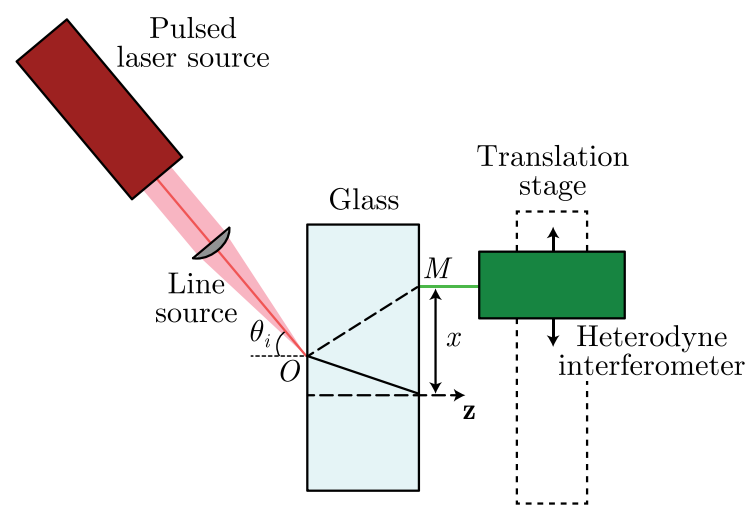

FIG. 6. (Color online) Experimental setup. 


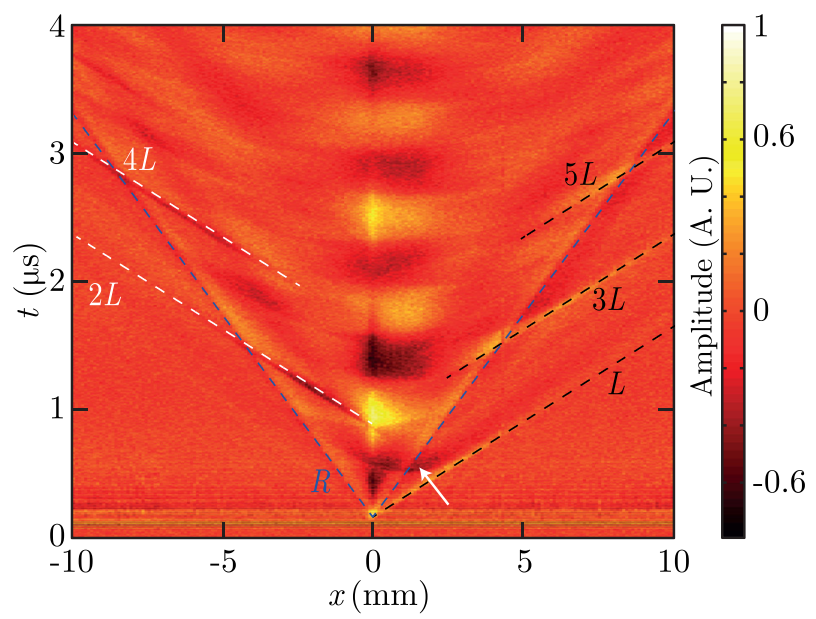

FIG. 7. (Color online) Normal displacement for $\varphi_{i}=50^{\circ}$ versus time $t$ and position $x$

at $x=0$ and are displayed for $t<4 \mu \mathrm{s}$. As expected, Rayleigh waves are detected on the rear surface of the window glass plate. The dashed line $R$ in Fig. 7 indicates the arrival time of the Rayleigh waves estimated from the sound velocities in Table I. The dashed lines, labeled $i L$ with $i \in\{1$ to 5$\}$, indicate the arrival time of the plane compressional waves with a propagation direction normal to the direction of refracted light propagation. Besides these predicted waves, the white arrow points out the shadow corresponding to the bulk compressional wavefront due to the thermoelastic generation at the front surface. The short time experimental results are in good agreement with the predicted displacement in Fig. 3.
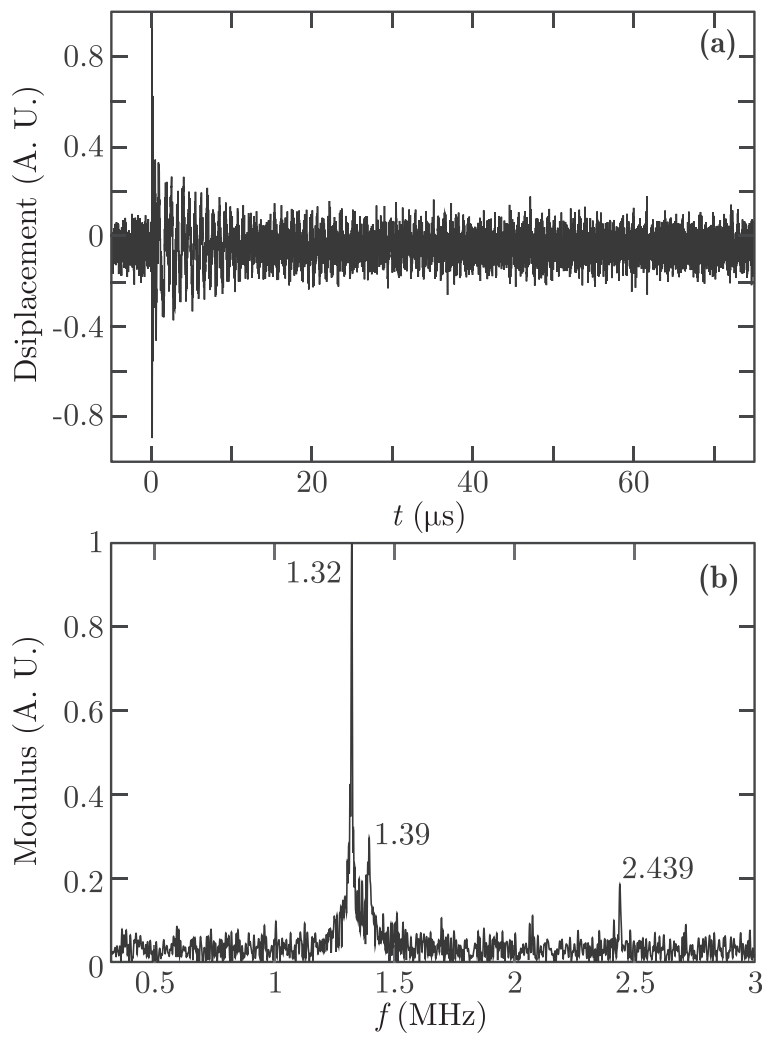

FIG. 8. (a) Normal displacement for $\varphi_{i}=50^{\circ}$ at position $x=2.2 \mathrm{~mm}$ as a function of time. (b) Time Fourier transform of the signal displayed in (a) using a temporal window: $t \in[5 ; 190] \mu \mathrm{s}$.
The experimental signals for $\varphi_{i}=50^{\circ}$ are now considered for longer times. Figure 8(a) displays the measured normal displacement at position $x=2.2 \mathrm{~mm}$ for times up to $75 \mu \mathrm{s}$. As mentioned previously, to detect both $\mathrm{ZGV}$ resonances with a single measurement, the detection point has indeed to be chosen such that the normal displacement is significant for both modes, which is the case for $x=2.2 \mathrm{~mm}$. The modulus of the time Fourier transform (FT) of this signal is shown in Fig. 8(b) using a temporal window: $t \in[5 ; 190] \mu \mathrm{s}$. The spectrum is composed of three peaks at 1.32, 1.39 , and $2.439 \mathrm{MHz}$. Referring to the dispersion curve in Fig. 2, the first and third frequencies correspond to the $S_{1} S_{2^{-}}$and the $A_{2} A_{3}-Z G V$ modes, respectively. The sharp $S_{1} S_{2}$-ZGV resonance is followed by a smaller resonance corresponding to the first extensional thickness resonance of the plate expected at $f=V_{L} / 2 d=1.391 \mathrm{MHz}$.

For each position of the interferometer, a temporal FT is applied using the temporal window $t \in[5 ; 190] \mu \mathrm{s}$, and displayed around the frequencies of both $\mathrm{ZGV}$ Lamb modes: $f_{S_{1} S_{2}}=1.32 \mathrm{MHz}$ and $f_{A_{2} A_{3}}=2.439 \mathrm{MHz}$. Figure 9 shows the FT modulus as a function of $x$ and for (a) $1.1 \mathrm{MHz}<f$ $<1.6 \mathrm{MHz}$ and for (b) $2.2 \mathrm{MHz}<f<2.7 \mathrm{MHz}$. Above each map, the upper frame displays the spatial distribution of the corresponding ZGV modes: experimental results (solid line) and the theoretical distribution (dashed line) discussed thereafter. In Fig. 9(a), the thickness resonance at $1.39 \mathrm{MHz}$ is quite uniform with respect to $x$, while the spatial distribution of the $S_{1} S_{2}-\mathrm{ZGV}$ mode at $f=1.32 \mathrm{MHz}$ shows five distinct lobes that confirm the interference mechanism of the $\mathrm{ZGV}$ resonance. As expected, the position of the main lobe
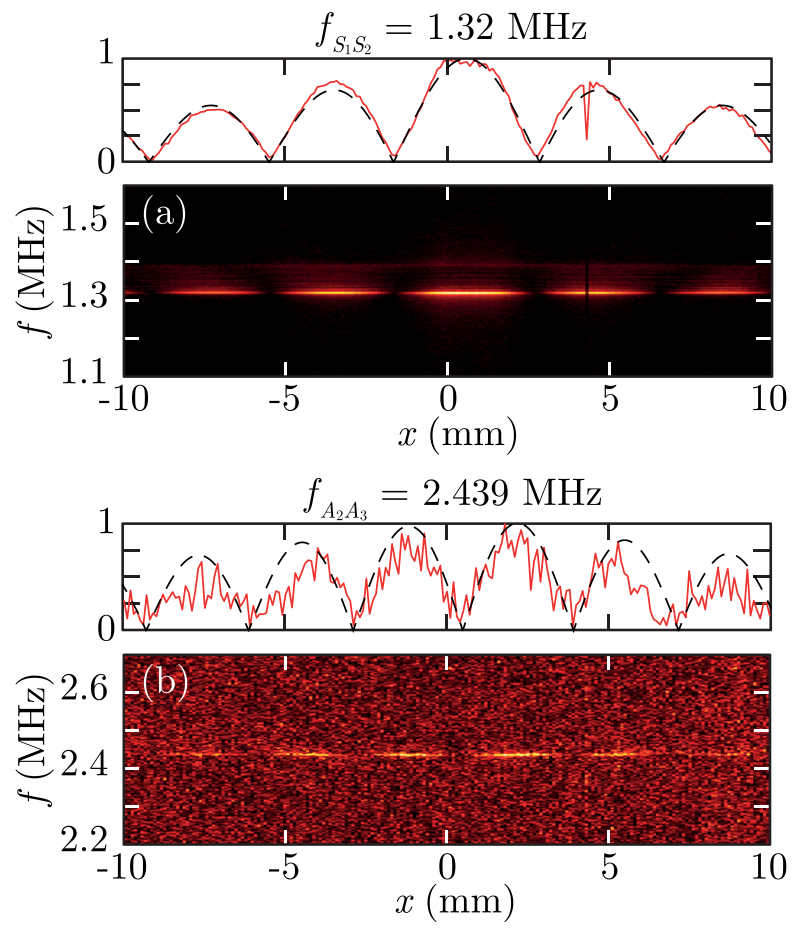

FIG. 9. (Color online) Fourier transform of the experimental signals versus position $x$ and frequency: (a) $f \in[1.1 ; 1.6] \mathrm{MHz}$, (b) $f \in[2.2 ; 2.7] \mathrm{MHz}$. Above (a) and (b), the upper frame displays the spatial distribution of the corresponding ZGV modes: experimental results (solid line) and theoretical distributions (dashed line). 
maximum for the $S_{1} S_{2}-\mathrm{ZGV}$ mode is shifted from $x=0$, and it corresponds to the predicted value $h \tan \varphi_{r}^{\text {real }}=0.61 \mathrm{~mm}$. In Fig. 9(b), the spatial distribution of the $A_{2} A_{3}-\mathrm{ZGV}$ mode at $f=2.439 \mathrm{MHz}$ is clearly visible although the signal to noise ratio at this frequency is one order of magnitude lower than for the $S_{1} S_{2}-Z G V$ mode. This confirms the prediction in Sec. III concerning the relative amplitude of the $A_{2} A_{3}-Z G V$ mode. The $A_{2} A_{3}-Z G V$ distribution exhibits six distinct lobes with one node close to the $x$ position of the axis of symmetry of the tilted volume source at $0.61 \mathrm{~mm}$.

With the laser beam focused to a line, the ZGV amplitude distribution should be theoretically a sinusoidal function of $x$. Yet the amplitude of lobes decreases with increasing the distance from the main lobe. This could be a consequence of mechanical damping or wave diffraction due to the finite length of the laser line. We now propose to account for the finite length of the source and to calculate the distribution $\Upsilon_{Z G V}(x)$ of ZGV modes. The integral formulation lies on the decomposition of the tilted volume source onto a continuous sum of point sources located along the laser line from $y=-L / 2$ to $y=L / 2$, where $L=14 \mathrm{~mm}$ stands for the length of the line. In this simplified formulation, only the contributions of two line sources at $\left(x=d \tan \varphi_{r}^{\text {real }}, z=0\right)$ and $(x=0, z=d)$ were considered, the distribution of the source along $z$ has not been taken into account. The mathematical expression of $\Upsilon_{Z G V}(x)$ that follows from the foregoing is

$$
\Upsilon_{Z G V}(x)=\int_{-L / 2}^{L / 2}\left\{J_{0}\left[k_{Z G V} \sqrt{\left(x-d \tan \varphi_{r}^{r e a l}\right)^{2}+\left(y-y_{S}\right)^{2}}\right] \pm \exp (-\beta d) J_{0}\left[k_{Z G V} \sqrt{x^{2}+\left(y-y_{S}\right)^{2}}\right]\right\} e^{-4 \ln 2\left(y_{S}^{2} / L^{2}\right)} \mathrm{d} y_{S} .
$$

This expression was obtained in three steps. First, and because a unique point source generates a ZGV mode with a Bessel distribution, the ZGV mode distribution due to a line of finite length $L$ is obtained by integrating the Bessel distribution along the line direction. Each point source is weighted by $\exp \left(-4 \ln 2 y_{S}^{2} / L^{2}\right)$ to account for the Gaussian distribution of the laser intensity. Second, to account for the laser absorption, the line source at $(x=0, z=d)$ is weighted by $\exp (-\beta d)$. Third, depending on the symmetry of the mode, the ZGV spatial distributions are obtained by summing $(S)$ or subtracting (A) the distributions of the two line sources. $\Upsilon_{Z G V}(x)$ is plotted in Fig. 9 (dashed lines). Good agreements are shown in Figs. 9(a) and 9(b) between theoretical and measured distributions. This demonstrates there is no need to take into account mechanical damping to predict the spatial distribution of ZGV modes.

We now compare the experimental results with the theoretical prediction in Fig. 5. To do so, for each $\varphi_{i}$, the temporal FT modulus is calculated and the spatial distributions of both ZGV modes are then obtained as previously explained. Then a spatial FT is applied to obtain the experimental

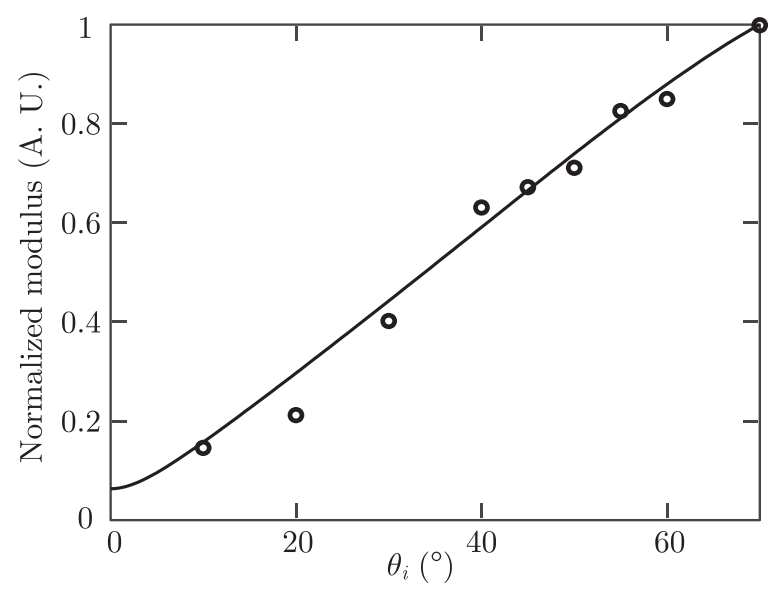

FIG. 10. Ratio of $S_{1} S_{2}$ and $A_{2} A_{3}$ resonance amplitudes as a function of the angle of incidence $\varphi_{i}$ : theory (solid line) and experiments (dots). values of $\left|\tilde{U}\left(f, k, \varphi_{i}\right)\right|$. To avoid any issue coming from changes in the laser power from experiment to experiment, we have calculated the ratio of the amplitude of the $S_{1} S_{2}$ - to that of the $A_{2} A_{3}-\mathrm{ZGV}$ resonance amplitudes. The normalized ratio is plotted with respect to $\varphi_{i}$ in Fig. 10. As experimental $\varphi_{i}$ are limited to $70^{\circ}$, the theoretical ratio is plotted for $\varphi_{i} \in$ $\left[0^{\circ} ; 70^{\circ}\right]$ and normalized to its maximum value too. The comparison is shown in Fig. 10. The good agreement between experimental and theoretical results demonstrates two major points: (i) the detection of both $\mathrm{ZGV}$ resonances with a single measurement is possible with the right detection location and (ii) increasing the angle of incidence increases the generation of the $A_{2} A_{3}-\mathrm{ZGV}$ mode in a glass plate. Then it is straightforward to find an optimal distance $x_{0}$ between the laser source and the interferometer where both $S_{1} S_{2^{-}}$and $A_{2} A_{3}-\mathrm{ZGV}$ modes can be best detected: for example, at $\varphi_{i}=50^{\circ}, x_{0}=2.2 \mathrm{~mm}$. Thus Poisson's ratio $\nu$ of the glass plate can be estimated knowing that $\nu$ is a monotonic function $g$ of the ratio of two ZGV frequencies: $\nu=g\left(f_{A_{2} A_{3}} / f_{S_{1} S_{2}}\right) .{ }^{32}$ The value deduced from the two resonance frequencies is $\nu=0.226$. The uncertainty $\Delta \nu$ depends on the width of the $\mathrm{ZGV}$ resonances. At $-3 \mathrm{~dB}$, the width is equal to $5 \mathrm{kHz}$ for both $\mathrm{ZGV}$ modes. Fitting the $\mathrm{ZGV}$ peaks reduces uncertainties on ZGV frequencies down to $\Delta f_{S_{1} S_{2}}$ $=\Delta f_{A_{2} A_{3}}=0.6 \mathrm{kHz}$. For $\nu=0.226$, the absolute uncertainty $\Delta \nu$ is equal to $m \Delta\left(f_{A_{2} A_{3}} / f_{S_{1} S_{2}}\right)$, where $m$ is the slope of $g$ and is equal to 0.79 at $\nu=0.226$. The absolute uncertainty on the Poisson's ratio is finally $\Delta \nu=0.001$. The measured value is in good agreement with the values $0.22-0.23$ reported for float glass. ${ }^{44,45}$

\section{CONCLUSION}

The absolute magnitude of elastic waves generated by laser in a low-absorbing material is lower than in a moderate- or high-absorbing material because less laser energy is deposited and the distribution of the acoustic energy of the source is different. The thermoelastic generation of $\mathrm{ZGV}$ 
Lamb modes in optically low-absorbing plates was investigated using a semi-analytical model. The analysis shows that wave fields generated by a surface or a volume source are very different.

In the case of a tilted volume thermoelastic source, it is shown that the generation of ZGV Lamb modes significantly depends on the angle of light incidence $\varphi_{i}$. The amplitude of the first symmetric $S_{1} S_{2}-\mathrm{ZGV}$ mode decays with $\varphi_{i}$, while the amplitude of the first antisymmetric $A_{2} A_{3}-\mathrm{ZGV}$ mode, very small at normal incidence, goes through a maximum for a large value of $\varphi_{i}$. At this angle, the amplitude of the $S_{1} S_{2^{-}}$ $\mathrm{ZGV}$ resonance remains about five times larger than the amplitude of the $A_{2} A_{3}-Z G V$ resonance; this allows precise determination of both resonance frequencies. Contrary to the case of a surface source, the antinodes of the normal displacement are not localized at the same position for symmetric and antisymmetric ZGV Lamb modes. In practice, the detection point must be shifted from the source center.

These theoretical predictions have been experimentally illustrated. Using a tilted volume thermoelastic source, the enhancement of the first antisymmetric ZGV Lamb mode while increasing the angle of light incidence was observed. The spatial distributions of the first symmetric $S_{1} S_{2}$ and antisymmetric $A_{2} A_{3}$ modes were measured; as predicted, the main lobe maxima for each mode are not at the same position on the plate surface. To detect both ZGV resonances with a single measurement, the detection point was chosen such that the normal displacement is significant for both modes. Using the property that the ratio of the first two ZGV frequencies is a monotonic function of the Poisson's ratio $\nu,{ }^{32}$ we obtained the value $\nu=0.226 \pm 0.001$ for a float glass plate at room temperature and without any knowledge of the sample thickness.

The technique we propose can be used to probe the Poisson's ratio in a wide range and with a good accuracy. Unlike conventional mechanical test, this technique is especially suitable to control the mechanical property homogeneity of a very large glass plate. Furthermore, such contactless measurements are possible under extreme conditions and are potentially interesting to follow phase transition.

\section{ACKNOWLEDGMENTS}

This work was supported by Saint-Gobain Recherche on the basis of a signed framework agreement with CNRS and by LABEX WIFI (Laboratory of Excellence ANR-10LABX-24) within the French Program Investments for the Future under Reference No. ANR-10 - IDEX-0001-02 PSL*.

${ }^{1}$ C. B. Scruby, R. J. Dewhurst, D. A. Hutchins, and S. B. Palmer, "Quantitative studies of thermally generated elastic waves in laserirradiated metals," J. Appl. Phys. 51, 6210-6216 (1980).

${ }^{2}$ J. A. Rogers, A. A. Maznev, M. J. Banet, and K. A. Nelson, "Optical generation and characterization of acoustic waves in thin films: Fundamentals and applications," Annu. Rev. Mater. Sci. 30, 117-157 (2000).

${ }^{3}$ J. D. Achenbach, "Laser excitation of surface wave motion," J. Mech. Phys. Sol. 51, 1885-1902 (2003), in Proceedings of a Symposium on Dynamic Failure and Thin Film Mechanics honoring Professor L. B. Freund.

${ }^{4}$ D. Hutchins and K. Lundgren, "A laser study of Rayleigh to bulk modeconversion at surface defects," in IEEE 1986 Ultrasonics Symposium, 1986, pp. 535-538.
${ }^{5}$ C. B. Scruby and L. Drain, Laser Ultrasonics: Techniques and Applications (Hilger, London, 1990), 462 pp.

${ }^{6}$ P. A. Doyle and C. M. Scala, "Near-field ultrasonic rayleigh waves from a laser line source," Ultrasonics 34, 1-8 (1996).

${ }^{7}$ D. C. Hurley, V. K. Tewary, and A. J. Richards, "Surface acoustic wave methods to determine the anisotropic elastic properties of thin films," Meas. Sci. Technol. 12, 1486-1494 (2001).

${ }^{8}$ S. Berezina, P. V. Zinin, D. Schneider, D. Fei, and D. A. Rebinsky, "Combining brillouin spectroscopy and laser-saw technique for elastic property characterization of thick DLC films," Ultrasonics 43, 87-93 (2004).

${ }^{9}$ F. Zhang, S. Krishnaswamy, and C. M. Lilley, "Bulk-wave and guidedwave photoacoustic evaluation of the mechanical properties of aluminum/ silicon nitride double-layer thin films," Ultrasonics 45, 66-76 (2006).

${ }^{10}$ C. Bescond, S. E. Kruger, D. Lévesque, R. S. Lima, and B. R. Marple, "In-situ simultaneous measurement of thickness, elastic moduli and density of thermal sprayed WC-Co coatings by laser-ultrasonics," J. Therm. Spray Technol. 16, 238-244 (2007).

${ }^{11}$ S. Mezil, N. Chigarev, V. Tournat, and V. Gusev, "All-optical probing of the nonlinear acoustics of a crack," Opt. Lett. 36, 3449-3451 (2011).

${ }^{12}$ R. M. Slayton, K. A. Nelson, and A. A. Maznev, "Transient grating measurements of film thickness in multilayer metal films," J. Appl. Phys. 90, 4392-4402 (2001).

${ }^{13}$ C. M. Hernandez, T. W. Murray, and S. Krishnaswamy, "Photoacoustic characterization of the mechanical properties of thin films," Appl. Phys. Lett. 80, 691-693 (2002).

${ }^{14}$ A. A. Maznev, A. Mazurenko, L. Zhuoyun, and M. Gostein, "Laser-based surface acoustic wave spectrometer for industrial applications," Rev. Sci. Instrum. 74, 667-669 (2003).

${ }^{15}$ C. Glorieux, J. D. Beers, E. H. Bentefour, K. Van de Rostyne, and K. A. Nelson, "Phase mask based interferometer: Operation principle, performance, and application to thermoelastic phenomena," Rev. Sci. Instrum. 75, 2906-2920 (2004).

${ }^{16}$ J. D. Choi, T. Feurer, M. Yamaguchi, B. Paxton, and K. A. Nelson, "Generation of ultrahigh-frequency tunable acoustic waves," Appl. Phys. Lett. 87, 081907 (2005).

${ }^{17}$ A. Bennis, A. M. Lomonosov, Z. H. Shen, and P. Hess, "Laser-based measurement of elastic and mechanical properties of layered polycrystalline silicon structures with projection masks," Appl. Phys. Lett. 88, 101915 (2006).

${ }^{18}$ When the optical absorption $\beta$ has negligible impact on the acoustic spectrum, $1 / \beta<c \tau$ with $c$ the sound velocity and $\tau$ the laser pulse duration, we consider the material to be of high-optical absorption. In the reverse case, we consider moderate-optical absorption. In the situation where $1 / \beta>d$, where $d$ stands for the sample thickness, the material is considered to be of low-optical absorption.

${ }^{19}$ D. H. Hurley, J. B. Spicer, R. J. Conant, and K. L. Telschow, "Determination of the optical absorption coefficient via analysis of lasergenerated plate waves," IEEE Trans. Ultrason. Ferroelectr. 44, 902-908 (1997).

${ }^{20} \mathrm{~K}$. L. Telschow and R. J. Conant, "Optical and thermal parameter effects on laser-generated ultrasound," J. Acoust. Soc. Am. 88, 1494-1502 (1990).

${ }^{21}$ B. Yuhai, P. Liwei, F. Qiping, Y. Yuping, and Y. Chongfu, "Experimental studies of directivity patterns of laser generated ultrasound in neutral glasses," Ultrasonics 33, 429-436 (1995).

${ }^{22}$ W. Yaping, S. Dufang, and H. Yulong, "Study of the directivity of laser generated ultrasound in solids," J. Appl. Phys. 83, 1207-1212 (1998).

${ }^{23}$ I. Arias and J. D. Achenbach, "Thermoelastic generation of ultrasound by line-focused laser irradiation," Int. J. Sol. Struct. 40, 6917-6935 (2003).

${ }^{24}$ D. Ségur, A. L. Shuvalov, B. Audoin, and Y. D. Pan, "Experimental and theoretical study of acoustic waves generated by a laser line pulse in an optically absorptive isotropic cylinder," J. Acoust. Soc. Am. 127, 181-185 (2010).

${ }^{25}$ S. Raetz, T. Dehoux, and B. Audoin, "Effect of laser beam incidence angle on the thermoelastic generation in semi-transparent materials," J. Acoust. Soc. Am. 130, 3691-3697 (2011).

${ }^{26}$ S. Raetz, T. Dehoux, M. Perton, and B. Audoin, "Acoustic beam steering by light refraction: Illustration with directivity patterns of a tilted volume photoacoustic source," J. Acoust. Soc. Am. 134, 4381-4392 (2013).

${ }^{27}$ D. Royer and E. Dieulesaint, Elastic Waves in Solids (Masson, Paris, 1999), Vol. 1, 374 pp. 
${ }^{28}$ J. D. Achenbach, Wave Propagation in Elastic Solids (North-Holland, American Elsevier, Amsterdam, 1973), 425 pp.

${ }^{29}$ C. Prada, O. Balogun, and T. W. Murray, "Laser-based ultrasonic generation and detection of zero-group velocity Lamb waves in thin plates," Appl. Phys. Lett. 87, 194109 (2005).

${ }^{30}$ D. Clorennec, C. Prada, D. Royer, and T. W. Murray, "Laser impulse generation and interferometer detection of zero group velocity Lamb mode resonance," Appl. Phys. Lett. 89, 024101 (2006).

${ }^{31}$ C. Prada, D. Clorennec, and D. Royer, "Local vibration of an elastic plate and zero-group velocity Lamb modes," J. Acoust. Soc. Am. 124, 203-212 (2008).

${ }^{32}$ D. Clorennec, C. Prada, and D. Royer, "Local and noncontact measurements of bulk acoustic wave velocities in thin isotropic plates and shells using zero group velocity Lamb modes," J. Appl. Phys. 101, 034908 (2007).

${ }^{33}$ T. Rouxel, H. Ji, T. Hammouda, and A. Moréac, "Poisson's ratio and the densification of glass under high pressure," Phys. Rev. Lett. 100, 225501 (2008).

${ }^{34}$ F. Decremps, L. Belliard, B. Perrin, and M. Gauthier, "Sound velocity and absorption measurements under high pressure using picosecond ultrasonics in a diamond anvil cell: Application to the stability study of alpdmn," Phys. Rev. Lett. 100, 035502 (2008).

${ }^{35}$ S. M. Nikitin, N. Chigarev, V. Tournat, A. Bulou, D. Gasteau, B. Castagnede, A. Zerr, and V. E. Gusev, "Revealing sub-m and m-scale textures in h2o ice at megabar pressures by time-domain Brillouin scattering," Sci. Rep. 5, 9532 (2015).

${ }^{36}$ R. J. Angel, J. M. Jackson, H. J. Reichmann, and S. Speziale, "Elasticity measurements on minerals: A review," Eur. J. Mineral. 21, 525-550 (2009).
${ }^{37}$ J. B. Spicer, A. D. W. McKie, and J. W. Wagner, "Quantitative theory for laser ultrasonic waves in a thin plate," Appl. Phys. Lett. 57, 1882-1884 (1990).

${ }^{38}$ D. R. França and A. Blouin, "All-optical measurement of in-plane and out-of-plane young's modulus and Poisson's ratio in silicon wafers by means of vibration modes," Meas. Sci. Technol. 15, 859-868 (2004).

${ }^{39}$ L.-P. Lefebvre, A. Blouin, S.-M. Rochon, and M. Bureau, "Elastic response of titanium foams during compression tests and using laserultrasonic probing," Adv. Eng. Mater. 8, 841-846 (2006).

${ }^{40}$ P. A. Mante, J. F. Robillard, and A. Devos, "Complete thin film mechanical characterization using picosecond ultrasonics and nanostructured transducers: Experimental demonstration on $\mathrm{SiO}_{2}$," Appl. Phys. Lett. 93, 071909 (2008).

${ }^{41}$ T. Takimura, N. Hata, S. Takada, and T. Yoshino, "Determination of mechanical properties of porous silica low- $k$ films on si substrates using orientation dependence of surface acoustic wave," Jpn. J. Appl. Phys. 47, 5400-5403 (2008)

${ }^{42}$ O. Balogun, G. Cole, R. Huber, D. Chinn, T. Murray, and J. Spicer, "High-spatial-resolution sub-surface imaging using a laser-based acoustic microscopy technique," IEEE Trans. Ultrason. Ferroelectr. Freq. Control 58, 226-233 (2011).

${ }^{43}$ X. Shan, X. Xiao, and Y. Liu, "Determination of Young's modulus and Poisson's ratio of nanoporous low- $k$ thin film by laser-generated surface acoustic waves," Adv. Sci. Lett. 4, 1230-1234 (2011).

${ }^{44}$ G. N. Greaves, A. L. Greer, R. S. Lakes, and T. Rouxel, "Poisson's ratio and modern materials," Nat. Mater. 10, 823-837 (2011).

${ }^{45} \mathrm{O}$. Goodman and B. Derby, "The mechanical properties of float glass surfaces measured by nanoindentation and acoustic microscopy," Acta Mater. 59, 1790-1799 (2011). 